Latent class analysis (LCA) was used to identify classes of MHC, and children were assigned to the class they had the highest probability of belonging to. We investigated associations between MHC classes and child's physical health (healthy weight, overweight or obese, based on measured heights and weights, using International Obesity Task Force cut-offs; maternal report of unintentional injuries since age 7 [none, 1, 2+]) and poor mental well-being (low self-esteem [SE] and low life-satisfaction [LS] reported by the child; maternal report of child's emotional problems [EP], all dichotomised at the bottom decile of scores). Relative risk ratios (aRRR, 95\% CI) and odds ratios (aORs, 95\% CI) were used to examine three-category and binary outcomes respectively, adjusting for potential confounding. Survey weights accounted for sample design and attrition. Analyses were undertaken in Stata/SE 13.1.

Results Four classes were identified (ranging from high to low $\mathrm{MHC})$ : "High learning skills and high prosocial behaviour" (37\%), "Moderate learning skills and high prosocial behaviour" (36\%), "Moderate learning skills and moderate prosocial behaviour" (19\%), and "Low learning skills and moderate prosocial behaviour" (8\%).

Risks of 2 +injuries were raised in "Moderate learning and high prosocial behaviour" (aRRR: 1.4 [95\%CI:1.2-1.7]) and "Low learning skills and moderate prosocial behaviour" (aRRR: 1.4 [95\%CI:1.1-1.9]) as compared to "High learning and high prosocial behaviour". Associations with MHC were absent or weak for single injury, overweight and obesity.

Compared to "High learning skills and high prosocial behaviour", odds of poor mental well-being were elevated for children from all other classes, with highest odds for "Low learning skills and moderate prosocial behaviour" (SE: aOR: 2.9 [95\%CI:2.3-3.6]; EP: aOR: 4.2 [95\%CI:3.4-5.1]; LS: aOR: 3.0 [95\%CI:2.4-3.7]).

Conclusion Lower MHC, using a composite measure developed in a representative sample of UK children, was associated with injuries, low self-esteem and life-satisfaction, and emotional problems, but not overweight and obesity. Identifying and promoting MHC at the population-level may provide an opportunity to improve health in children and young people.

\section{P42 "UNSEEN INJURIES": INVISIBILITY AND MENTAL ILLNESS IN THE ENGLISH WELFARE SYSTEM}

KJ Pybus* , K Pickett, C Lloyd. Department of Health Sciences, University of York, York, UK

\subsection{6/jech-2017-SSMAbstracts. 144}

Background Research focusing on the impact of current welfare reform in England has identified that people with mental illnesses experience pronounced difficulties when accessing social security compared to those with other health conditions. This includes a disproportionate risk of being sanctioned, problematic assessments resulting from a system designed to focus on physical capabilities and a greater likelihood of being viewed as a fraudulent claimant. The impact of individual policies is useful evidence, however the existence of disadvantage across several aspects of this system may imply the presence of underlying mechanisms which need to be understood if further marginalisation is to be prevented. The perspective of individuals with mental illness has been largely omitted from this discussion and may provide useful insights into these topics. The current study therefore aimed to explore the lived experiences of people with mental illness accessing housing and income benefits in England.

Methods Participants were recruited through five organisations offering support with social circumstances to people with mental illness in Leeds, England. Semi-structured interviews were completed with adults $(n=11)$ accessing housing and income benefits, living independently in the community. Convenience sampling in conjunction with organisation staff was employed to recruit participants. The sample was comprised of participants with the following mental health issues: depression $(\mathrm{n}=8)$, anxiety $(\mathrm{n}=5)$, post-traumatic stress disorder $(n=1)$, bi-polar affective disorder $(n=1)$, psychosis $(n=2)$, borderline personality disorder $(n=1)$ and substance misuse $(n=2)$ with several instances of co-morbidity. Data was interrogated using a six stage thematic analysis approach.

Results Accessing housing and income benefits can be problematic for people with mental illness due to the 'unseen' nature of these conditions. Barriers included being unable to provide evidence of illness to obtain financial support and the level of system flexibility in recognising the impact of mental health needs. Alongside this, participants encountered stigma from family, friends and the wider community rooted in the perceived validity of mental illness as a reason for claiming benefits. These themes were present across different types of mental health condition.

Conclusion These findings suggest that to create a social security system designed to be effective for people with a mental illness; changes are needed to the way in which mental illness is understood, assessed and monitored in this context. This study used a diverse but small sample in a localised setting and further research is needed to confirm these findings.

\section{P43 THE ASSOCIATION BETWEEN DEPRESSION AND SUBSEQUENT HYPERTENSION-A SYSTEMATIC REVIEW AND META-ANALYSIS}

R Prigge*, CA Jackson. Usher Institute of Population Health Sciences and Informatics, The University of Edinburgh, Edinburgh, UK

\subsection{6/jech-2017-SSMAbstracts. 145}

Background The possible causal association between depression and cardiovascular disease might be partly explained by an increased risk of hypertension. Several epidemiological studies have investigated the role of depression in the development of hypertension but the evidence is inconclusive. A previous systematic review of these studies has a number of shortcomings, including inappropriate pooling of different effect measures and lack of inclusion of all relevant studies. Additional primary studies have also been published since this earlier review was completed. Our aim was to identify, critically appraise, and synthesise evidence on the association between depression and subsequent hypertension.

Methods We performed a systematic electronic search in PsycINFO, Medline, and EMBASE to identify cohort or longitudinal studies reporting on the risk of hypertension among participants with versus without depressive symptoms and/or clinical depression. We restricted our search to articles published in English. We extracted information on study characteristics, methodology, and results using customised data extraction forms and assessed study quality using the SIGN checklist for cohort studies. We used Stata 14 to perform 